\title{
Traits and states in mindfulness meditation
}

\author{
Yi-Yuan Tang, Britta K. Hölzel and Michael I. Posner
}

In our Review (The neuroscience of mindfulness meditation. Nat. Rev. Neurosci. 16, $213-$ $225(2015))^{1}$, we described recent research into the neural mechanisms and consequences of mindfulness meditation. In their comment on our article (What is being studied as mindfulness meditation? Nat. Rev. Neurosci. http:// dx.doi.org/10.1038/nrn.2015.6) ${ }^{2}$, Wheeler, Arnkoff and Glass pointed out the importance of differentiating between dispositional mindfulness (also known as trait mindfulness) and deliberate (intentional) mindfulness meditation $^{2}$. Their suggestion is consistent with our point of view with regard to individual differences in mindfulness meditation ${ }^{1,3}$. Although we restricted our Review to studies that aimed to investigate mindfulness as an intentional practice $^{1}$, pre-existing differences in dispositional mindfulness might have affected the findings described.

So far, relatively little is known about how differences in dispositional mindfulness might influence brain processing and the effective practice of mindfulness. However, a number of studies within the past decade have investigated the neural correlates of dispositional mindfulness and have identified some functional and structural brain areas involved ${ }^{4-9}$.

Dispositional mindfulness is usually assessed through self-report questionnaires, such as the Mindful Attention Awareness $S_{\text {Scale }}{ }^{10}$, the Kentucky Inventory of Mindfulness Skills ${ }^{11}$ or Five Facet Mindfulness Qu estionnaire $^{12}$. The use of these questionnaires comes with specific challenges and limitations, which have been critically discussed ${ }^{13}$, and a recent review has concluded that the evidence to support the validity of these questionnaires is lacking ${ }^{12}$. It is therefore important to remember that what is interpreted as 'dispositional mindfulness' is what these questionnaires assess.
It is known that people differ in their attitude towards and practice of mindfulness meditation $^{1,3}$. Growing evidence has indicated that mindfulness practice induces both state and trait changes: that is, it temporarily changes the condition of the brain and the corresponding pattern of activity or connectivity (state change), and it also alters personality traits following a longer period of practice. It had been traditionally assumed that personality traits are relatively stable entities, but more recent research demonstrates that personality, including disposition towards mindfulness, can change over time as a result of life experiences or through mindfulness practice ${ }^{14,15}$, suggesting that personality is itself flexible. Although this demonstrates that individuals can change the way that they feel, believe, and act, it also complicates the systematic investigation of the construct of 'dispositional mindfulness', as Wheeler, Arnkoff and Glass suggest ${ }^{2}$. Nevertheless, this recent evidence suggests that is will be important to assess dispositional mindfulness at different points during studies investigating the effects of mindfulness meditation and distinguish it from intentional mindfulness.

Individual differences in personality are likely to contribute to how people respond to and benefit from mindfulness practice, in the same way that differences in brain function and structure, genetic predisposition, life experiences and environmental factors do. However, more empirical studies are needed to establish a definitive effect of these factors on mindfulness. More longitudinal, randomized, and actively controlled studies with larger sample sizes should deepen our understanding of how people with different personality traits practice effectively.

Yi-Yuan Tang is at the Department of Psychological Sciences, Texas Tech University, Lubbock, Texas 79409, USA.
Britta K. Hölzel is at the Department of Neuroradiology, Technical University of Munich, 81675 Munich, Germany and Massachusetts General Hospital, Charlestown, Massachusetts 02129, USA.

Michael I. Posner is at the Department of Psychology, University of Oregon, Eugene, Oregon 97403, USA.

Correspondence to Y.-Y.T. yiyuan.tang@ttu.edu

1. Tang, Y.-Y., Hölzel, B. K. \& Posner, M. I. The neuroscience of mindfulness meditation. Nat. Rev Neurosci. 16, 213-225 (2015).

2. Wheeler, M. S., Arnkoff, D. B. \& Glass, C. R. What is being studied as mindfulness meditation? Nat. Rev. Neurosci. http://dx.doi.org/10.1038/nrn.2015.6 (2016).

3. Tang, Y.-Y. \& Posner, M. I. Tools of the trade: theory and method in mindfulness neuroscience. Soc. Cogn. Affect Neurosci. 8, 118-120 (2013).

4. Lu, H. et al. The brain structure correlates of individual differences in trait mindfulness: a voxel-based morphometry study. Neuroscience 272, 21-28 (2014).

5. Kong, F. et al. Brain regions involved in dispositional mindfulness during resting state and their relation with well-being. Soc. Neurosci. Sep. 25, 1-13 (2015).

6. Creswell, J. D., Way, B. M., Eisenberger, N. I. $\&$ Lieberman, M. D. Neural correlates of dispositional mindfulness during affect labeling Psychosomat. Med. 69, 560-565 (2007).

7. Modinos, G., Ormel, J. ¿ Aleman, A. Individual differences in dispositional mindfulness and brain activity involved in reappraisal of emotion. Soc. Cogn. Affect. Neurosci. 5, 369-377 (2010).

8. Shaurya Prakash, R., De leon, A. A., Klatt, M., Malarkey, W. \& Patterson, B. Mindfulness disposition and default-mode network connectivity in older adults. Soc. Cogn. Affect. Neurosci. 8, 112-117 (2013).

9. Taren, A. A., Creswell, J. D. \& Gianaros, P. J. Dispositional mindfulness co-varies with smaller amygdala and caudate volumes in community adults. PLoS One 8, e64574 (2013).

10. Brown, K. W. \& Ryan, R. M. The benefits of being present: mindfulness and its role in psychological wellbeing. J. Pers. Soc. Psychol. 84, 822-848 (2003).

11. Baer, R. A., Smith, G. T. \& Allen, K. B. Assessment of mindfulness by self-report: the Kentucky inventory of mindfulness skills. Assessment 11, 191-206 (2004).

12. Park, T., Reilly-Spong, M. \& Gross, C. R. Mindfulness: A systematic review of instruments to measure an emergent patient reported outcome (PRO). Qual. Life Res. 22, 2639-2659 (2013).

13. Grossman, P. Defining mindfulness by how poorly I think I pay attention during everyday awareness and other intractable problems for psychology's (re) invention of mindfulness: comment on Brown et al. Psychol. Assess. 23, 1034-1040 (2011).

14. Nyklīček, I., van Beugen, S. \& Denollet, J. Effects of mindfulness-based stress reduction on distressed (type D) personality traits: a randomized controlled trial. J. Behav. Med. 36, 361-370 (2013).

15. Shapiro, S. L., Brown, K. W., Thoresen, C. \& Plante, T. G. The moderation of mindfulness-based stress reduction effects by trait mindfulness: results from a randomized controlled trial. J. Clin. Psychol. 67, 267-277 (2011).

\section{Acknowledgements}

This work was supported by the US Office of Naval Research.

\section{Competing interests statement}

The authors declare no competing interests. 